\title{
Cognitive changes in older women after urogynaecological surgery
}

\author{
Sonja Brandner', Celine Aeberhard ${ }^{2}$, Michael D. Mueller ${ }^{3}$, Annette Kuhn \\ ${ }^{1}$ Frauenzimmer Bern AG, Bern, Switzerland \\ ${ }^{2}$ Rheumatology, Departement of Medicine, Inselspital, Switzerland \\ ${ }^{3}$ Urogynaecology, Department of Gynaecology, Frauenklinik, Inselspital Bern, Switzerland
}

\begin{abstract}
Objectives: The need for pelvic floor surgery will increase with an aging population in the future. Aim of this prospective study was to evaluate the evolution of cognitive function in elderly women after urogynaecological surgery.

Material and methods: Between 2010 and 2014, 51 female patients 70 years and older who underwent urogynaecological surgery participated in this study. Geriatric and urogynaecological assessment were performed before and six weeks after surgery, including the Mini-COG test, the clock-drawing test, a depression scale, an activities of daily living questionnaire, and the German pelvic floor questionnaire.

Results: Mean age was 77 years (range 70-91). Overall, 15 women were operated for incontinence, 31 for prolapse, and five for miscellaneous reasons. Only two (3.9\%) of the 51 women developed postoperative delirium. Abnormal cognitive findings increased from preoperatively $15.7 \%$ to $39.2 \%$ six weeks after surgery (odds ratio $3.4,95 \%$ confidence interval 1.3 to $8.7, p<0.001$ ). There were no statistically significant pre-post differences in activities of daily living and depression scores. Pelvic floor function indices improved significantly

Conclusion: This study shows an overall decline of cognitive function in a vulnerable group of elderly women during the short-term postoperative period. Postoperative cognitive dysfunction (POCD) after surgery has been described for other types of non-cardiac surgery but has been understudied in urogynaecological surgery. These patients need interdisciplinary management to prevent or minimize adverse effects of surgery on cognitive function.
\end{abstract}

Key words: cognitive function; urogynaecological interventions; pelvic floor function; activities of daily living; dementia; postoperative complications

Ginekologia Polska 2018; 89, 11: 587-592

\section{BACKGROUND}

The worldwide population is aging. For 2050, it is expected that one third of the Swiss population will be over 65 years old, whereof $12 \%$ will be older than 80 years [1]. Urogynaecological problems, particularly prolapse and incontinence, are increasing with age. As a consequence, we expect an increase of urogynaecological interventions over the next decades. Based on Data from 2010 Kirby et al [2] calculated an increase in requirement of urogynaecological interventions of $35 \%$ for 2030.

There is a paucity of safety data analysing urogynaecological operations in the frail and elderly. Most randomized studies exclude this group of women because of suggested higher morbidity [3]. Whilst $16 \%$ of all women over 70 years are operated for stress urinary incontinence, $3.8 \%$ of elderly patients only are represented in studies as it has been shown by Morse [3]. Riemsma et al identified only four trials for his review, which reported cure rates in incontinence therapy in the elderly population. All of these studies where referring to drug therapy. No trial was found about cure rates in incontinence or prolapse surgery in elderly [4]. Data analysing abdominal interventions have shown that complication rates between $7-20 \%$ are higher in patients 70 years and older depending on their general state of health and their current activities of daily living (ADL) [5]. Frailty, impaired cognitive function and depression [6] are risk factors for higher morbidity. Concerning pelvic floor surgery a review showed a complication rate between 15.5 and $33 \%$ in this 
older group of patients. These complications include urinary tract infections, fever as well as postoperative bleeding with the necessity of blood transfusion [7].

Cognition is critical for functional independence as people age, including whether people are able to live independently, manage finances, take medications correctly, and drive safely. Cognitive abilities often decline with age [8].

Postoperative disorientation has been shown between $4.6 \%$ [9] of cases in a urogynecological group of patients and $7.4 \%$ respectively in a number of patients undergoing major gynaecological interventions. However, there is a lack of data in urogynaecological patients [10].

\section{OBJECTIVES}

Aim of this prospective study was to evaluate the evolution of cognitive function among older women after urogynaecological surgical interventions for incontinence and prolapse.

\section{METHODS}

Ethical committee gave the permission for this prospective cohort study (KEK-BE 030/10) and patients gave written and oral consent to participate. Between 2010 and 2014 all female patients 70 years and older at admission being admitted for urogynaecological interventions in the gynaecological department of University Hospital of Bern who did not already participate in a parallel ongoing study were asked to participate in this study.

Patients were evaluated one day before surgery and six weeks after. Type and time of surgery, type of anaesthesia, blood loss and intraoperative complications were noted as well as hospital stay, short term complications and occurrence of delirium or confusion. All operations were performed by two experienced urogynaecologists $(S B, A K)$ in a tertiary referral setting.

Cognitive function was measured using the Mini-Cog test and the clock-drawing test performed on the day before and in the postop visit 6 weeks after surgery. The test before surgery was done by the resident doctor at the preoperative visit. Postoperative testing was done by the two surgeons. For the Mini-Cog, the tested person is asked to repeat three words, draw a clock with all numbers and hands, and then to repeat the three words. For the clock drawing test, the person is then asked to write down the time of the clock as if it were in a schedule for trains or in a TV (Television) guide. Scoring of the Mini Cog test was done based on the algorithm described by Borson et al. [11], based on a combined assessment of the clock and recall of the three words. The clock drawing test was analysed separately using two different scoring methods. We calculated the score as described by Wang et al. [12] which is based on seven criteria, and has been shown to be sensitive to change over time.
A cut-off point of $<3$ on the Mini-Cog test has been validated for dementia screening.

Activities of daily living ( $A D L$ ) were measured using a combined measure of basic and instrumental activities of daily living, based on the methods described by Katz [13] and Fillenbaum [14]. It ranges from 10 to 40 , with higher scores denoting higher levels of dependency. For the purpose of a dichotomous analysis, impairment in ADL was defined as one or more self-reported limitation in activities of daily living.

Risk for depression was measured using the five-item Geriatric Depression score [15]. It ranges from 0 to 5, with higher values denoting a higher risk for depression. A score of $\geq 2$ has been shown to be a valid cut-off for identifying people at increased risk for depression.

Postoperative diagnosis of delirium was made clinically.

Patients filled in the validated pelvic floor questionnaire (German pelvic floor questionnaire [16]) and a visual analogue scale (VAS) from 10 - no satisfaction at all to 0 - the highest possible satisfaction before and six weeks after surgery to determine quality of life (QOL). The exact wording was "How bothersome is your urogynaecological condition currently?"The Global pelvic floor function score was calculated according to the method described by Baessler and Kemkensteffen [16]. The global score ranges from 0 to 40, with higher scores denoting higher levels of impairment. It is composed of four function sub scores (bladder, intestinal, prolapse, and sexual), each sub score ranging from 0 to 10 .

In addition, as objective outcome measures Pelvic Organ Prolapse Quantification (POP-Q) in prolapse patients and cough and PAD Test in the incontinence group were performed before and six weeks after surgery.

\section{Statistical analysis}

Primary outcome was the score of the mini-Cog test 6 week after surgery, secondary outcomes were quality of life as determined by the German pelvic floor questionnaire and VAS, POP-Q values as measurement of prolapse and PAD test as objective measurement of incontinence.

We described the study population by mean values, standard deviation (SD), frequency ( $\mathrm{n}$ ) and percentage (\%). We defined change as the score difference between follow-up and baseline. We tested whether the change of a score was equal to zero (null hypothesis of no change) using a t-test [17]. As sensitivity analysis we used the Wilcoxon test [18]. We assessed the odds of being depressed, limited in ADL, and demented at follow-up using logistic regression models, reporting Odds Ratios (OR) and 95\% confidence intervals $(\mathrm{Cl})$.

Power calculation was performed using Graph Pad version 2.0 for Windows (San Diego, USA). Assuming that the true difference between means before and after surgery of 3.24 in the MiniCOg test, the system calculated that 
Table 1. Functional scores derived from geriatric assessment at baseline and at 6-week follow-up

\begin{tabular}{|l|l|l|l|l|}
\hline Function & Baseline & Follow-Up & Difference (95\% CI) & p-value \\
\hline Cognition score & $3.9( \pm 2.1)$ & $2.9( \pm 2.2)$ & $-1.0(-1.6,-0.41$ & 0.002 \\
\hline Activities of daily living score & $11.4( \pm 2.4)$ & $11.5( \pm 3.0)$ & $0.1(-0.3,0.5)$ & 0.58 \\
\hline Depression score & $0.6( \pm 0.9)$ & $0.5( \pm 0.8)$ & $-0.1(-0.4,0.1)$ & 0.39
\end{tabular}

Values are mean $\pm \mathrm{SD}$ — standard deviation; $\mathrm{Cl}$ — confidence interval values; cognition scores — lower scores denote higher risk; activities of daily living and depression scores - higher scores denote higher risk; difference defined as mean score difference between follow-up and baseline; $\mathrm{p}$-value from t-test

Table 2. Geriatric risk factors derived from geriatric assessment at baseline and at 6-week follow-up

\begin{tabular}{|l|l|l|l|l|}
\hline Risk factor & Baseline & Follow-Up & Odds Ratio (95\% Cl*) & p-value \\
\hline Possible cognitive impairment & $8(15.7 \%)$ & $20(39.2 \%)$ & $3.4(1.3,8.7)$ & $<0.001$ \\
\hline Impairment in activities of daily living & $18(35.3 \%)$ & $16(31.4 \%)$ & $0.8(0.4,1.9)$ & 0.67 \\
\hline Possible depression & $9(17.7 \%)$ & $4(7.8 \%)$ & $0.4(0.1,1.5)$ & 0.19 \\
\hline
\end{tabular}

*Odds ratio: baseline finding is reference value. $\mathrm{Cl}$ denotes confidence interval; Values are mean \pm SD — standard deviation

\begin{tabular}{|l|l|l|l|}
\hline \multicolumn{4}{|l}{ Table 3. Pelvic floor function scores* at baseline and at 6-week follow-up } \\
\hline Function & Baseline & Follow-Up & Difference (95\% CI) \\
\hline Bladder function & $4.03( \pm 1.80)$ & $2.42( \pm 1.82)$ & $-1.60(-2.14,-1.06)$ \\
\hline Bowel function & $2.12( \pm 1.37)$ & $1.74( \pm 1.35)$ & $-0.35(-0.65,-0.04)$ \\
\hline Prolapse function & $3.11( \pm 2.75)$ & $0.35( \pm 0.81)$ & $-2.76(-3.50,-2.02)$ \\
\hline Sexual function & $0.17( \pm 0.60)$ & $0.13( \pm 0.68)$ & $-0.04(-0.15,0.08)$ \\
\hline Global pelvic floor function & $9.42( \pm 3.63)$ & $4.62( \pm 3.23)$ & $-4.77(-5.90,-3.65)$ \\
\hline
\end{tabular}

Values are mean \pm SD — standard deviation; $\mathrm{Cl}$ — confidence interval; lower scores denote better function; difference defined as mean score difference between follow-up and baseline; $p$-values from t-test

$\mathrm{n}=50$ patients are necessary to detect statistical significant difference with a power of $90 \%$.

\section{RESULTS}

Between February 2010 and March 2014 a total of 62 women were eligible for recruitment. Five patients refused to give informed consent for participation in the study. Six women were lost to follow-up leaving 51 patients in total. Mean age was 77 years (range 70-91).

Fifteen incontinence operations (incontinence group) and 31 prolapse operations (prolapse group) were performed, three interventions due to mesh complications and two because of complications caused by suburethral slings (miscellaneous group). In detail, we performed eleven suburethral slings, one colposuspension, one vaginal fistula repair, two Botox injections for incontinence, 28 vaginal prolapse operations whereof six included vaginal hysterectomy and two included Mesh insertion and three laparoscopic sacrocolpopexies for prolapse, three vaginal Mesh excisions for erosions and two vaginal urethrolyses for chronic urinary retention.

Twenty nine interventions were performed under general anaesthesia, 19 under spinal anaesthesia and three under local anaesthetics using lidocaine $2 \% 10-20 \mathrm{~mL}$.
Operating time was 52.5 minutes (range 20-330 minutes), hospital stay four days (1-21 days) and blood loss $100 \mathrm{~mL}(0-800 \mathrm{~mL})$.

Table 1 summarizes the results of cognition scores, daily living and depression. Cognitive function deteriorated significantly.

There were no statistically significant differences in the pre- and postoperative activity of daily living indices. Overall, two (3.9\%) of the 51 patients with delirium and confusion had to be referred to a nursing home postoperatively.

Table 2 shows the results of geriatric assessment using dichotomous definitions of the assessment scores.

Abnormal cognitive findings increased from preoperatively $15.7 \%$ to $39.2 \%$ six weeks after surgery (odds ratio $3.4,95 \%$ $\mathrm{Cl} 1.3$ to $8.7, \mathrm{p}<0.001$ ). In contrast the proportion of patients with possible impairment in activities of daily living or with possible depression did not change from baseline to follow-up.

Pelvic floor function as determined by the German Pelvic Floor Questionnaire is summarized in Table 3.

Bladder and bowel function, prolapse and global pelvic floor function sub scores improved significantly postoperatively. Sexual function did not change, but this sub score was not sensitive to change because 43 ( $84.3 \%$ of the 51 ) women reported that they did not engage in sexual activity. 
Table 4. Change estimates (6-week follow-up versus baseline) of score function, sensitivity analyses based on Wilcoxon test

\begin{tabular}{|l|l|l|}
\hline Domain & Difference $(95 \% \mathrm{Cl})$ & p-value \\
\hline Cognition score & $-1.50(-2.50,-0.50)$ & 0.004 \\
\hline Activities of daily living score & $0.00(-1.00,1.50)$ & 0.729 \\
\hline Depression score & $-0.50(-1.00,0.50)$ & 0.301 \\
\hline Bladder function score & $-1.56(-2.11,-1.00)$ & $<0.001$ \\
\hline Intestinal function & $-0.29(-0.59,0.00)$ & 0.077 \\
\hline Prolapse function score & $-3.67(-4.67,-3.00)$ & $<0.001$ \\
\hline Sexual function score & $-0.48(-1.43,0.95)$ & 0.528 \\
\hline Global pelvic floor function score & $-4.67(-5.88,-3.48)$ & $<0.001$ \\
\hline
\end{tabular}

Difference defined as median score difference between follow-up and baseline; $\mathrm{Cl}$ - confidence interval; $\mathrm{p}$-value from Wilcoxon test

Table 4 summarizes the sensitivity analyses with change estimates of the geriatric assessment and pelvic score function scores based on Wilcoxon test. Results did not change using these test procedures.

We also conducted specific analyses in subgroups of patients. In patients of the incontinence group ( $n=15)$, Pad test improved significantly from a median of 34 grams (4-103 grams) preoperatively to 6 grams postoperatively (054 grams; $p<0.001$, t-test). In the patients who underwent prolapse surgery $(n=28)$ POP-Score measuring the most distal point improved significantly from a median of $+3 \mathrm{~cm}$ (range $+1-+12 \mathrm{~cm})$ to $-1 \mathrm{~cm}(-3$ to $+1 \mathrm{~cm} ; \mathrm{p}<0.001)$ postoperatively, and patient satisfaction (VAS score) improved significantly from an initial median of $9(6-10)$ to a median of $2(0-4, p<0.001)$.

\section{DISCUSSION}

The current study shows a substantial deterioration of cognitive function six weeks postoperatively in older women after urogynaecological surgery. To our knowledge, this is one of very few studies analyzing the evolution of cognitive function in gynaecological surgery [19]. One study performed geriatric assessment before and six months after urogynaecological surgery, and did not find evidence for cognitive decline. However, this study included a high-functioning group of women with only 2/31 women exhibiting an abnormal cognitive score as measured by the Mini Cog test [20]. In a review, Menard [21] reports post-operative disorientation in $4.6 \%$ of women after urogynaecological surgery. However, clinically diagnosed cognitive dysfunction likely underestimates true cognitive dysfunction, as measured by a validated cognitive test.

Some recent studies in fact do confirm that the prevalence of cognitive impairment is relatively high in these persons. Trowbridge [22] describes that $40 \%$ of patients older than 80 years perceived themselves as suffering from memory loss; and Erekson [19] detected a rate of $21 \%$ patients screening positive for dementia in a group of $150 \mathrm{pa}-$ tients who suffering from pelvic floor disorders. Our study identified $16 \%$ of patients with possible dementia, which is similar to the data by Erekson and lower as described by Trowbridge but in the latter study, the patients were slightly older than in our study.

Several studies have shown postoperative cognitive dysfunction (POCD) in patients after other types of surgery [23]. Many of those studies were performed in cardiac and orthopedic units. In these operations, high blood loss and potentially oxygen deficit intraoperatively may play a role; in cardiac surgery, a general pre-existent vascular alteration may additionally affect cognitive function.

Several publications have shown a relation between intraoperative blood loss and reduction of cognitive function in elderly. Menard [21] published the necessity of blood transfusion in $12 \%$ of patients. In the current study, there was only one intervention with significant blood loss of $800 \mathrm{~mL}$, so we do not consider this as an important aspect in the current study.

Another issue of potential cognitive impairment is the type of anaesthesia [23, 24]. In both studies, there was no correlation of decline in cognitive function with the type of anaesthesia. Type of anaesthesia and surgery seems to play a subsidiary role $[23,25,26]$ particularly in the long term follow up [27]. As functional reserve of all organs is reduced with age, this applies also to the brain reserve. The current study is too small to perform sub-analyses of different types of anaesthetics.

Jankowski showed that preoperative reduced cognitive function and reduced verbal memory predicts postoperative delirium [28].

One pathophysiological explanation of the worsening of cognitive function in this elderly group of patients is that any surgery, trauma, hospital stay or anaesthesia may cause inflammatory processes in the brain. Macrophages enter the hypocampus through a damaged blood brain barrier and cause death of microglia cells [29]. Several groups have indeed shown that due to stress or trauma a complex neuroinflammatory process is induced which leads in decline of cognitive function or delirium by of death of microglia cells in the hippocampus region $[19,29]$. Elderly women and concomitant multipharmacy (especially anticholinergic drugs) seem to lead to a greater vulnerability to those effects.

We do not know whether those processes are reversible or not. It would have been interesting to evaluate the cognitive function half a year later. There are long term studies ongoing showing that the decline in cognitive function is reversible [30].

Pelvic floor function including bladder and bowel function did improve in this elderly group of patients as 
described before in many studies with the exception of sexual function. Due to the small number of sexually active patients, we cannot draw any conclusion concerning the development of sexual function from the current study.

The weakness of this study included heterogeneous urogynaecologic interventions and not a single entity; however, we feel that we still get a valuable result looking at these various interventions. Another weakness is that the study contains a small number of patients only with no control group. Recruiting patients was difficult, and roughly $10 \%$ of patients did decline to participate in this study. We can only speculate why patients denied participation because this has not been investigated; however, during the course of this study, we got the impression that there was an obvious fear of being diagnosed with cognitive impairment whilst participating. However, power calculation determined that the number of 50 patients would be enough to receive statistical valuable results. In the current study, follow-up was six weeks only, and we have no data on the long-term sequelae. The strength of this study was the use of validated tools, the prospective character of the study and the high mean age of 77 years.

We conclude that assessment of frailty and physiological reserves including cognitive function in the preoperative evaluation for urogynecological surgeries is important. Results should be involved in preoperative counselling and our decision making process. Further long-term data for the security and global function in daily life of those patients will be needed.

An interdisciplinary approach between urogynaecologist, geriatrician and anaesthetist to identify patients at risk for delirium and to avoid declining cognitive function would possibly be the ideal setting for the elderly including preoperative assessment by the geriatrician. Costs arising from a setting like that can be a critical issue.

\section{CONCLUSION}

Elderly women are at high risk for adverse cognitive outcome after urogynaecological surgery. These patients may benefit from an interdisciplinary approach between urogynaecologist, geriatrician and other involved disciplines in the pre- and postoperative period. Systematic geriatric assessment before and after surgery will inform clinicians about risk for adverse outcomes and need for focused early intervention.

\section{Acknowledgements}

Sonja Brandner, Frauenzimmer Bern AG, Switzerland - Project development, data collection, writing manuscript; Celine Aeberhard, Rheumatology, Departement of Medicine, Inselspital, Switzerland - data collection, literature review, feasibility study ; Annette Kuhn, Urogynaecology, Depart- ment of Gynaecology, Frauenklinik, Inselspital Bern, Switzerland - Project development, data analyses, statistical analyses, writing manuscript; Michael D. Mueller, Department of Gynaecology, Frauenklinik, Inselspital Bern, Switzerland — literature review, intellectual input

Thanks to André Moser for his help in the statistical part and also special thanks to Professor Andreas Stuck, Head of the Department of Geriatrics, Bern University Hospital and Spital Netz Bern and University of Bern, Switzerland - for his important inputs in the project development, data analyses and interpretation.

\section{Declarations}

Ethics approval and consent to participate: Ethical committee of the Kanton Bern gave the permission for this prospective cohort study (KEK-BE 030/10). After oral and written information, all patients provided oral and written consent to participate in this study using the appropriate consent form as determined by the local ethical committee and according to the current Swiss law.

Funding: no funding for this study

\section{Conflict of interests}

None of the coauthors have a conflict of interest.

\section{REFERENCES}

1. BFS Szenarien zur Bevölkerungsentwicklung der Schweiz 2010-2060 Statistik der Schweiz. Neuchâtel, 01.07.2010.

2. Kirby A, Luber K, Menefee S. An update on the current and future demand for care of pelvic floor disorders in the United States. American Journal of Obstetrics and Gynecology. 2013; 209(6): 584.e1-584.e5, doi: 10.1016/j.ajog.2013.09.011.

3. Morse AN, Labin LC, Young SB, et al. Exclusion of elderly women from published randomized trials of stress incontinence surgery. Obstet Gynecol. 2004; 104(3): 498-503, doi: 10.1097/01.AOG.0000136484.37027.79, indexed in Pubmed: 15339759.

4. Riemsma R, Hagen S, Kirschner-Hermanns R, et al. Can incontinence be cured? A systematic review of cure rates. BMC Med. 2017; 15(1): 63, doi: 10.1186/s12916-017-0828-2, indexed in Pubmed: 28335792.

5. Pofahl WE, Pories WJ. Current status and future directions of geriatric general surgery. J Am Geriatr Soc. 2003; 51 (7 Suppl):S351-S354, indexed in Pubmed: 12823667.

6. Makary M, Segev D, Pronovost $P$, et al. Frailty as a Predictor of Surgical Outcomes in Older Patients. Journal of the American College of Surgeons. 2010; 210(6): 901-908, doi: 10.1016/j.jamcollsurg.2010.01.028.

7. Gerten KA, Richter HE. Pelvic floor surgery in the older woman. Clin Obstet Gynecol. 2007; 50(3): 826-843, doi: 10.1097/GRF.0b013e3180de4702, indexed in Pubmed: 17762429.

8. Murman D. The Impact of Age on Cognition. Seminars in Hearing. 2015 36(03): 111-121, doi: 10.1055/s-0035-1555115.

9. Toglia MR, Nolan TE. Morbidity and mortality rates of elective gynecologic surgery in the elderly woman. Am J Obstet Gynecol. 2003; 189(6): 1584-7; discussion 1587, indexed in Pubmed: 14710073.

10. Newman S, Stygall J, Hirani S, et al. Postoperative cognitive dysfunction after noncardiac surgery: a systematic review. Anesthesiology. 2007 106(3): 572-590, indexed in Pubmed: 17325517.

11. Borson S, Scanlan J, Brush M, et al. The Mini-Cog: a cognitive, vital signs' measure for dementia screening in multi-lingual elderly. International Journal of Geriatric Psychiatry. 2000; 15(11): 1021-1027, doi: 10.1002/1099-1166(200011)15:11<1021::aid-gps234>3.0.co;2-6.

12. Wang $P$, Shi $L$, Zhao Q, et al. Longitudinal Changes in Clock Drawing Test (CDT) Performance before and after Cognitive Decline. PLoS ONE. 2014; 9(5): e97873, doi: 10.1371/journal.pone.0097873. 
13. Katz S, Ford AB, Moskowitz RW, et al. Studies of Illness in the Aged. JAMA. 1963; 185(12): 914, doi: 10.1001/jama.1963.03060120024016.

14. Fillenbaum GG. Screening the elderly. A brief instrumental activities of daily living measure. J Am Geriatr Soc. 1985; 33(10): 698-706, indexed in Pubmed: 4045087.

15. Hoyl MT, Alessi CA, Harker JO, et al. Development and testing of a five-item version of the Geriatric Depression Scale. J Am Geriatr Soc. 1999; 47(7): 873-878, indexed in Pubmed: 10404935.

16. Baessler K, Kempkensteffen C. Validierung eines umfassenden Beckenboden-Fragebogens für Klinik, Praxis und Forschung. Gynäkologisch-geburtshilfliche Rundschau. 2009; 49(4): 299-307, doi: 10.1159/000301098.

17. Regression Methods in Biostatistics: Linear, Logistic, Survival, and Repeated Measures Models (Statistics for Biology and Health). Springer 2012.

18. Hollander M, Wolfe DA, Chicken E. Nonparametric Statistical Methods. 2015, doi: 10.1002/9781119196037.

19. Zhang $\mathrm{X}$, Dong $\mathrm{H}$, Li N, et al. Activated brain mast cells contribute to postoperative cognitive dysfunction by evoking microglia activation and neuronal apoptosis. Journal of Neuroinflammation. 2016; 13(1), doi: 10.1186/s12974-016-0592-9.

20. Erekson EA, Fried TR, Martin DK, et al. Frailty, cognitive impairment, and functional disability in older women with female pelvic floor dysfunction. Int Urogynecol J. 2015; 26(6): 823-830, doi: 10.1007/s00192-014-2596-2, indexed in Pubmed: 25516232

21. Menard JP, Mulfinger C, Estrade JP, et al. Chirurgie du prolapsus génital de la femme âgée de plus de 70 ans : revue de la littérature. Gynécologie Obstétrique \& Fertilité. 2008;36(1):67-73, doi:10.1016/j.gyobfe.2007.04.025.

22. Trowbridge $E$, Kim D, Barletta K, et al. Prevalence of positive screening test for cognitive impairment among elderly urogynecologic pati- ents. American Journal of Obstetrics and Gynecology. 2016; 215(5): 663. e1-663.e6, doi: 10.1016/j.ajog.2016.06.012.

23. Ancelin ML, Roquefeuil Gde, Scali J, et al. Long-Term Post-Operative Cognitive Decline in the Elderly: The Effects of Anesthesia Type, Apolipoprotein E Genotype, and Clinical Antecedents. Journal of Alzheimer's Disease. 2010; 22(s3): S105-S113, doi: 10.3233/jad-2010-100807.

24. Richter HE, Redden DT, Duxbury AS, et al. Pelvic floor surgery in the older woman: enhanced compared with usual preoperative assessment. Obstet Gynecol. 2005; 105(4): 800-807, doi: 10.1097/01. AOG.0000154920.12402.02, indexed in Pubmed: 15802408.

25. Cole MG. Delirium in Elderly Patients. American Journal of Geriatric Psychiatry. 2004; 12(1): 7-21, doi: 10.1176/appi.ajgp.12.1.7.

26. Steinmetz J, Rasmussen LS. The elderly and general anesthesia. Minerva Anestesiol. 2010; 76(9): 745-752, indexed in Pubmed: 20820153.

27. Evered L, Scott D, Silbert B, et al. Postoperative Cognitive Dysfunction Is Independent of Type of Surgery and Anesthetic. Anesthesia \& Analgesia. 2011; 112(5): 1179-1185, doi: 10.1213/ane.0b013e318215217e.

28. Jankowski C, Trenerry M, Cook D, et al. Cognitive and Functiona Predictors and Sequelae of Postoperative Delirium in Elderly Patients Undergoing Elective Joint Arthroplasty. Anesthesia \& Analgesia. 2011; 112(5): 1186-1193, doi: 10.1213/ane.0b013e318211501b.

29. Terrando N, Eriksson L, Ryu JK, et al. Resolving postoperative neuroinflammation and cognitive decline. Annals of Neurology. 2011; 70(6): 986-995, doi: 10.1002/ana.22664.

30. Davis N, Lee M, Lin A, et al. Postoperative Cognitive Function Following General Versus Regional Anesthesia. Journal of Neurosurgical Anesthesiology. 2014; 26(4): 369-376, doi: 10.1097/ana.0000000000000120. 